\section{Mortalidade perinatal por sífilis congênita: indicador da qualidade da atenção à mulher e à criança}

\author{
Perinatal mortality due to congenital syphilis: \\ a quality-of-care indicator for women's \\ and children's healthcare
}

\author{
1 Secretaria Municipal \\ de Saúde do Rio de Janeiro, \\ Rio de Janeiro, Brasil. \\ 2 Escola Nacional de Saúde \\ Pública, Fundação Oswaldo \\ Cruz, Rio de Janeiro, Brasil. \\ Correspondência \\ V. Saraceni \\ Secretaria Municipal \\ de Saúde do Rio de Janeiro. \\ Rua Cupertino Durão 219, \\ bloco B, apto. 404 \\ Rio de Janeiro, $R J$ \\ 22441-030, Brasil. \\ valeria@alternex.com.br
}

\begin{abstract}
Syphilis is a persistent cause of perinatal mortality in Rio de Janeiro, Brazil, where this study was performed using data from the mortality data system and investigational reports for fetal and neonatal deaths, mandatory in municipal maternity hospitals. From 1996 to 1998, 13.1\% of fetal deaths and 6.5\% of neonatal deaths in municipal maternity hospitals were due to congenital syphilis. From 1999 to 2002, the proportions were $16.2 \%$ and $7.9 \%$, respectively. For the city of Rio de Janeiro as a whole from 1999 and 2002, the proportions were $5.4 \%$ of fetal deaths and $2.2 \%$ of neonatal deaths. The perinatal mortality rate due to congenital syphilis remains stable in Rio de Janeiro, despite efforts initiated with congenital syphilis elimination campaigns in 1999 and 2000. We propose that the perinatal mortality rate due to congenital syphilis be used as an impact indicator for activities to control and eliminate congenital syphilis, based on the investigational reports for fetal and neonatal deaths. Such reports could be extended to the surveillance of other avoidable perinatal disease outcomes.
\end{abstract}

Infant Mortality; Congenital Syphilis; Women's Health; Child Welfare
Valéria Saraceni 1

Maria Helena Freitas da Silva Guimarães ${ }^{1}$ Mariza Miranda Theme Filha 1

Maria do Carmo Leal 2

\section{Introdução}

A sífilis é causa de grande morbidade na vida intra-uterina, levando a desfechos negativos da gestação em mais de 50,0\% dos casos, tais como, aborto, nati e neomortalidade e complicações precoces e tardias nos nascidos vivos 1,2 .

A sífilis congênita ainda ocupa espaço entre as causas básicas de óbitos infantis, sobretudo entre as perdas fetais. Gust et al. 3 , estudando a letalidade específica por sífilis congênita nos Estados Unidos no período de 19921998, encontraram 760 natimortos entre 14.627 casos. Em um estudo de coorte prospectivo na Tanzânia, 51,0\% dos casos de natimortos estavam relacionados à sífilis materna 4 . Na Federação Russa, vem se registrando um aumento da incidência da doença, e um estudo de 850 grávidas identificou 544 casos que resultaram em 26,0\% de óbitos fetais ou neonatais 5. Para os anos de 1996 a 1998, no Município do Rio de Janeiro, Brasil, foram contabilizados 88 casos de sífilis congênita entre os 670 casos de natimortos ocorridos nas maternidades municipais $(13,1 \%)$ e 43 casos de sífilis congênita $(6,5 \%)$ entre 664 óbitos neonatais 6 . Esses estudos, ocorridos em lugares com situações epidemiológicas tão distintas, demonstram a persistente participação da sífilis na mortalidade perinatal.

A mortalidade fetal tem sido subdimensionada no Brasil, embora compartilhe muitos aspectos da sua ocorrência com o óbito neonatal 
precoce 7,8 . A sua notificação é tão precária que os dados de Indicadores Básicos para a Saúde (IDB 2002) da Rede Interagencial de Informações para a Saúde (RIPSA) mostram taxas de mortalidade perinatal registradas apenas para os Estados do Espírito Santo, Rio de Janeiro, São Paulo, Paraná, Santa Catarina, Rio Grande do Sul e Mato Grosso do Sul, variando de 16,1 (Santa Catarina/1997) a 31,2 (Rio de Janeiro/1999) por mil nascimentos vivos 9 .

A sífilis congênita se insere no quadro de causa perinatal evitável, pois é possível fazer o diagnóstico e proceder ao tratamento efetivo na gestação. No Município do Rio de Janeiro, aonde a mortalidade infantil vem caindo seguidamente, as causas perinatais estiveram nos primeiros lugares entre as causas básicas de óbito no período compreendido entre 1999 e 2002. A sífilis congênita contribuiu de forma significativa nesse grupo, ocupando o quinto lugar entre os natimortos.

Este estudo pretende descrever a tendência da mortalidade perinatal por sífilis congênita no Município do Rio de Janeiro, entre os anos de 1999 e 2002, estabelecendo um paralelo com as campanhas de eliminação da sífilis congênita realizadas no município em 1999 e 2000, descritas em outro local 10. O estudo pretende, também, estabelecer as bases para introduzir a mortalidade perinatal específica por sífilis congênita como indicador de impacto das ações programáticas para o controle da mortalidade perinatal no Município do Rio de Janeiro.

\section{Métodos}

O período perinatal compreende o intervalo entre a 22a semana de gestação e o sétimo dia de vida após o nascimento. Na ausência de informação sobre a idade gestacional, a Organização Mundial da Saúde (OMS) recomenda o uso do peso acima de $500 \mathrm{~g}$ como ponte de corte inicial para esse período. Quanto à mortalidade, compõe-se da natimortalidade somada à mortalidade neonatal precoce 11 . O cálculo da taxa de mortalidade perinatal tem, no seu numerador, o número de óbitos ocorridos dentro do período perinatal e, no denominador, todos os nascidos vivos acrescidos dos natimortos para o ano em questão, multiplicada por mil 12 .

As bases de dados aqui analisadas foram cedidas pela Coordenação de Programas de Epidemiologia da Secretaria Municipal de Saúde do Rio de Janeiro (SMS-RJ). As informações sobre os óbitos vieram do Sistema de Informações de Mortalidade (SIM) e das Fichas de Notificação e Investigação de Óbitos Fetais e Neonatais.

Desde 1998, as nove maternidades municipais do Rio de Janeiro vêm notificando, no período de 24 horas, a ocorrência de todos os óbitos neonatais. A investigação desses óbitos e, também, dos óbitos fetais têm sido feita no período máximo de trinta dias. As fichas de investigação são preenchidas, no caso dos óbitos fetais, pelos obstetras e, no caso dos óbitos neonatais, pelos pediatras, ou por profissionais do serviço de epidemiologia hospitalar. Esta base de dados foi comparada com o SIM para verificar se todos os casos se encontravam representados em ambas.

Os óbitos fetais e neonatais das mães residentes no Município do Rio de Janeiro foram analisados ano a ano, e, como a distribuição dos atributos maternos e fetais eram semelhantes, os anos foram analisados em conjunto. A mortalidade por sífilis congênita foi estudada em diversos componentes, a saber, natimortalidade, mortalidade neonatal precoce e perinatal.

A variável peso ao nascer foi estratificada em < 1.000g; 1.000-1.499g; 1.500-1.999g; 2.000$2.499 \mathrm{~g}$ e $2.500 \mathrm{~g}$ e mais 13 . Para algumas análises, considerou-se apenas o critério dicotômico de baixo peso ao nascer 14 . Foi considerado parto prematuro aquele ocorrido antes de 37 semanas de gestação.

Das fichas de investigação de óbito fetal e neonatal, procurou-se obter outros dados relativos à sífilis materna, como a titulação do VDRL no parto, na tentativa de estabelecer associação entre os níveis de VDRL materno e o resultado no concepto.

$\mathrm{Na}$ análise estatística, utilizou-se o teste de $\chi^{2}$, com significância estatística de 0,95 para verificar a homogeneidade das proporções. Analisou-se o comportamento temporal dos óbitos perinatais e da taxa de mortalidade perinatal geral e por sífilis congênita através do coeficiente de determinação $\mathrm{R}^{2}$, por regressão linear simples.

\section{Resultados}

Foram analisados 7.309 óbitos perinatais (4.215 fetais e 3.094 neonatais precoces) de filhos de mães residentes no Município do Rio de Janeiro, no período de 1999 a 2002. Destes, 292 (4,0\%) tiveram como causa básica a sífilis congênita, 
sendo $222(5,3 \%)$ fetais e $70(2,3 \%)$ neonatais precoces.

Na Tabela 1, pode observar-se um declínio no número absoluto de óbitos perinatais por ano $\left(R^{2}=0,951 ; p=0,049\right)$, o que não se repete nos óbitos perinatais relacionados à sífilis congênita $\left(\mathrm{R}^{2}=0,088 ; \mathrm{p}=0,704\right)$. A participação proporcional da sífilis congênita entre os óbitos fetais é significativamente maior do que entre os óbitos neonatais precoces $(\mathrm{p}=0,0000)$.

Na Tabela 2, pode verificar-se que a taxa de mortalidade perinatal por mil nascimentos foi de 20,2; 19,3; 17,9 e 18,6, respectivamente, para os anos de 1999, 2000, 2001 e 2002. A taxa de mortalidade perinatal específica para sífilis congênita no mesmo período foi de 0,8; 0,8; 0,6 e 0,9 . Os coeficientes de determinação das taxas não foram significativos, sugerindo estabilidade de ambas.

Na Tabela 3, encontram-se as características maternas e de nascimento dos casos de óbitos perinatais, com e sem sífilis congênita. Pode observar-se que as mães dos casos de sífilis congênita eram mais jovens e tinham menor escolaridade $(\mathrm{p}=0,0000)$. A prematuridade e o baixo peso ao nascer também foram maiores entre os óbitos perinatais por sífilis congênita do que no outro grupo $(\mathrm{p}=0,0000)$.

A análise estratificada do peso ao nascer mostrou que, nos grupos abaixo de $1.499 \mathrm{~g}$, não foi diferente entre os conceptos com e sem sífilis congênita. Nas faixas intermediárias entre 1.500-2.499g, as proporções foram significativamente maiores no grupo com sífilis congênita. Todavia, nos óbitos com peso igual ou maior do que $2.500 \mathrm{~g}$, as maiores proporções ficaram para o grupo sem sífilis congênita $(p=0,0000)$.

Contabilizaram-se, nesse último ano, $65 \mathrm{ca}$ sos de óbitos fetais de puérperas residentes no Município do Rio de Janeiro, investigados atra- vés das fichas, cuja ocorrência se deu em mães com as seguintes características: $29,2 \%$ eram adolescentes, $47,7 \%$ freqüentaram o atendimento pré-natal, e 50,8\% tinham história de abortos anteriores. A informação da titulação do VDRL no momento do parto estava ausente em $41,5 \%$ dessas mulheres. Nas que tinham informação, verificou-se que, em $83,1 \%$ dos casos, a titulação do VDRL materno foi igual ou superior a $1 / 16$. Entre os natimortos, $89,2 \%$ nasceram com peso menor do que $2.500 \mathrm{~g}$, e $93,8 \%$, antes de 37 semanas de gestação.

Dos 22 óbitos neonatais precoces ocorridos por sífilis congênita em 2002, verificou-se que $19,0 \%$ das mães eram adolescentes, $70,0 \%$ tinham história de abortamento prévio, e 47,6\% estavam inseridas no pré-natal. OVDRL no parto apresentava títulos inferiores a 1/16 em $44,4 \%$, mas $16,0 \%$ não tinham o preenchimento desse campo. O baixo peso ao nascer ocorreu em $95,5 \%$ dos casos, e a prematuridade, em 90,5\%. A Tabela 4 apresenta esses dados e a comparação entre eles, evidenciando que não há diferença entre os óbitos fetais e neonatais precoces quanto às variáveis analisadas, excetuando-se os títulos de VDRL igual ou superiores a 1/16, mais freqüentes entre os óbitos fetais.

Ao comparar-se os dados do SIM com os das fichas de investigação, percebeu-se uma grande concordância entre as duas fontes de informação. Entretanto, 9 dos 87 óbitos estudados não teriam sido identificados se a busca tivesse contemplado apenas a causa básica do óbito. As regras básicas de classificação do óbito definidas pela OMS 11 priorizam alguns diagnósticos em detrimento da sífilis congênita, levando-a a aparecer como causa contribuinte, mas não determinante da morte. Dois casos de óbitos fetais não foram encontrados no SIM, embora as fichas de investigação existissem. Is-

Tabela 1

Óbitos fetais, neonatais precoces e perinatais de mães residentes no Município do Rio de Janeiro, Brasil, 1999/2002.

\begin{tabular}{|c|c|c|c|c|c|c|}
\hline \multirow[t]{2}{*}{ Ano } & \multicolumn{2}{|c|}{$\begin{array}{l}\text { Óbito fetal } \\
(n=4.215)\end{array}$} & \multicolumn{2}{|c|}{$\begin{array}{l}\text { Óbito neonatal precoce } \\
\qquad(\mathrm{n}=3.094)\end{array}$} & \multicolumn{2}{|c|}{$\begin{array}{c}\text { Óbito perinatal } \\
(n=7.309)\end{array}$} \\
\hline & Geral & $\begin{array}{l}\text { Por sífilis } \\
\text { congênita }\end{array}$ & Geral & $\begin{array}{l}\text { Por sífilis } \\
\text { congênita }\end{array}$ & Geral & $\begin{array}{l}\text { Por sífilis } \\
\text { congênita }\end{array}$ \\
\hline 1999 & 1.236 & 57 & 879 & 22 & 2.115 & 79 \\
\hline 2000 & 1.124 & 67 & 802 & 12 & 1.926 & 79 \\
\hline 2001 & 923 & 42 & 713 & 14 & 1.636 & 56 \\
\hline 2002 & 932 & 56 & 700 & 22 & 1.632 & 78 \\
\hline
\end{tabular}

Fonte: Sistema de Informações sobre Mortalidade, 1999/2002. 
so pode dever-se a problemas de interpretação da letra do investigador na ficha, gerando nomes errôneos que não se consegue identificar posteriormente.

\section{Discussão}

As características das mães com sífilis descritas neste estudo são muito semelhantes às encontradas nas mães que participaram das campanhas de eliminação da sífilis congênita nos anos de 1999 e 2000 15. São mulheres com piores condições sócio-econômicas, mais jovens e com menor escolaridade. Recentemente, Lago et al. 16 descreveram o mesmo perfil materno em Porto Alegre.

A concentração de óbitos por sífilis congênita no período fetal, dentro da mortalidade perinatal, permitiu mostrar as profundas desigualdades na ocorrência desses óbitos no $\mathrm{Mu}$ nicípio do Rio de Janeiro, onde a queda progressiva da mortalidade na infância vinha atenuando as diferenças nas taxas de mortalidade infantil entre os grupos sociais 17.

O impacto da sífilis congênita na viabilidade fetal é demonstrado pela diferença significativa entre a proporção de casos de óbitos fetais comparada com os neonatais precoces. A sífilis materna já foi associada a um risco dez vezes maior de ocorrência de perda fetal 18. A ocorrência de maior proporção de mulheres com títulos de VDRL iguais ou superiores a 1/16 entre os óbitos fetais corrobora outros estudos que mostraram um efeito deletério dos estágios iniciais da sífilis sobre os fetos 19,20,21.

No Brasil, apenas alguns Estados da Federação disponibilizam informação sobre a mortalidade perinatal, e assim sendo, pouco se conhece sobre a magnitude da mortalidade fetal tardia ${ }^{9}$. Se a situação encontrada no Município do Rio de Janeiro aplicar-se ao restante do país, é preocupante o subdimensionamento que pode estar ocorrendo na ocorrência da sífilis congênita, principalmente porque tem se mostrado que as áreas nacionais que detêm pior qualidade de informação nos Sistemas de Informação em Saúde têm também piores indicadores de condição de vida e saúde 22 .

As doenças sexualmente transmissíveis (DST), por lidarem com as questões ligadas à sexualidade, tendem a ser encobertas, tornarem-se invisíveis. No caso da sífilis, pela remissão espontânea das lesões das fases primária e secundária, seguida de longo período de latência clínica, fica difícil a identificação de portadores, levando Carrara 23 (p. 46) a dizer que “...se a sífilis se propaga tão amplamente é em grande parte por ser invisivel".
Tabela 2

Taxa de mortalidade perinatal por mil nascimentos, geral e por sífilis, de mães residentes no Município do Rio de Janeiro, Brasil, 1999/2002.

\begin{tabular}{ccc}
\hline Ano & $\begin{array}{l}\text { Taxa de mortalidade } \\
\text { perinatal geral }\end{array}$ & $\begin{array}{l}\text { Taxa de mortalidade perinatal } \\
\text { por sífilis congênita }\end{array}$ \\
\hline 1999 & 20,2 & 0,75 \\
2000 & 19,3 & 0,79 \\
2001 & 17,9 & 0,61 \\
2002 & 18,6 & 0,89 \\
\hline
\end{tabular}

Fonte: Sistema de Informações sobre Mortalidade/

Sistema de Informações sobre Nascimentos, 1999/2002.

Tabela 3

Distribuição percentual dos óbitos perinatais por sífilis congênita e outras causas segundo algumas características maternas e dos conceptos. Município do Rio de Janeiro, Brasil, 1999/2002.

\begin{tabular}{|c|c|c|c|}
\hline Características & $\begin{array}{l}\text { Por sífilis } \\
\text { congênita } \\
(n=292)\end{array}$ & $\begin{array}{l}\text { Outras causas } \\
(n=7.017)\end{array}$ & p-valor \\
\hline \multicolumn{4}{|c|}{ Idade materna (anos) } \\
\hline$<20$ & 44,9 & 22,6 & 0,0000 \\
\hline 20 e mais & 65,1 & 77,4 & \\
\hline \multicolumn{4}{|c|}{$\begin{array}{l}\text { Escolaridade materna } \\
\text { (anos) }\end{array}$} \\
\hline Até 7 & 81,6 & 56,9 & 0,0000 \\
\hline 8 e mais & 18,4 & 43,1 & \\
\hline \multicolumn{4}{|l|}{ Prematuridade } \\
\hline Sim & 82,1 & 69,1 & 0,0000 \\
\hline Não & 17,9 & 30,9 & \\
\hline \multicolumn{4}{|c|}{ Peso ao nascer $(\mathrm{g})$} \\
\hline$<1.000$ & 29,1 & 33,8 & 0,0969 \\
\hline $1.000-1.499$ & 20,4 & 17,4 & 0,1651 \\
\hline $1.500-1.999$ & 19,6 & 13,2 & 0,0020 \\
\hline $2.000-2.499 \mathrm{~g}$ & 17,5 & 11,4 & 0,0016 \\
\hline$>2.500$ & 13,5 & 24,1 & 0,0000 \\
\hline
\end{tabular}

Fonte: Sistema de Informações sobre Mortalidade, 1999/2002.

Essa invisibilidade pode perpassar também pelos serviços de saúde, como na definição da causa mortis. Na classificação da causa de óbito perinatal, as regras utilizadas para a mortalidade geral não se aplicam, sendo utilizados outros recursos para estabelecer a seqüência correta dos eventos que culminaram com o óbito, como a análise de causas múltiplas ${ }^{11}$. No Mu- 
Tabela 4

Distribuição percentual dos óbitos fetais e neonatais precoces por sífilis congênita segundo algumas características maternas e dos conceptos. Município do Rio de Janeiro, Brasil, 2002.

\begin{tabular}{|c|c|c|c|}
\hline & $\begin{array}{l}\text { Óbitos fetais } \\
(n=65)\end{array}$ & $\begin{array}{l}\text { Óbitos neonatais } \\
\qquad(\mathrm{n}=22)\end{array}$ & p-valor \\
\hline \multicolumn{4}{|c|}{ Idade materna (anos) } \\
\hline$<20$ & 29,2 & 19,0 & 0,3100 \\
\hline 20 e mais & 60,8 & 81,0 & \\
\hline \multicolumn{4}{|c|}{ Abortos anteriores } \\
\hline Sim & 50,8 & 70,0 & 0,1558 \\
\hline Não & 49,2 & 30,0 & \\
\hline \multicolumn{4}{|c|}{ Inserção no pré-natal } \\
\hline Sim & 47,7 & 47,6 & 0,8558 \\
\hline Não & 52,3 & 52,4 & \\
\hline \multicolumn{4}{|c|}{ Prematuridade } \\
\hline Sim & 93,8 & 90,5 & 0,9866 \\
\hline Não & 6,2 & 9,5 & \\
\hline \multicolumn{4}{|c|}{ Peso ao nascer $(\mathrm{g})$} \\
\hline$<2.500$ & 89,2 & 95,5 & 0,6553 \\
\hline$>2.500$ & 10,8 & 4,5 & \\
\hline \multicolumn{4}{|c|}{ VDRL materno } \\
\hline$<1 / 8$ & 16,9 & 44,4 & 0,0069 \\
\hline$>1 / 16$ & 83,1 & 55,6 & \\
\hline
\end{tabular}

Fonte: Fichas de Notificação e Investigação de Óbitos Fetais e Neonatais, Secretaria Municipal de Saúde do Rio de Janeiro, 2002. ção da titulação do VDRL materno no caso dos óbitos fetais.

As fichas de notificação de óbito fetal e neonatal têm sido utilizadas para a melhoria dos Sistemas de Informação em Saúde, tanto corrigindo as causas básicas do óbito, quanto notificando agravos. As altas taxas de incidência e de mortalidade perinatal por sífilis congênita no Município do Rio de Janeiro refletem esse cuidado, mas sabe-se que, ainda assim, as taxas estão sujeitas a uma subnotificação importante. As maternidades municipais são responsáveis por aproximadamente $50,0 \%$ dos partos do Município do Rio de Janeiro, mas notificaram mais de $90,0 \%$ de todos os casos conhecidos de sífilis congênita no período compreendido entre 1996 e 19986.

Entre os casos de óbitos fetais e neonatais precoces atendidos e notificados pelas maternidades municipais no ano de 2002, a sífilis congênita representou $16,2 \%$ dos óbitos fetais e 7,9\% dos neonatais precoces. A relevância desses achados fala por si mesma e mostra uma piora da situação em relação ao período de 1996-1998.

Agravos como a sífilis congênita quase sempre refletem questões relacionadas ao acesso e à utilização de serviços de saúde, atingindo prioritariamente a população mais desfavorecida 24 . No estudo de morbimortalidade perinatal realizado no Município do Rio de Janeiro, Leal et al. 25 verificaram que apenas $3,8 \%$ das mães desse estudo não realizaram pré-natal, e, das que realizaram, $71,8 \%$ foram admitidas no primeiro trimestre da gestação, e somente $3,0 \%$, no terceiro. Apesar do amplo acesso ao pré-natal e ao parto hospitalar, ainda se convive com uma baixa qualidade da atenção prestada 26 .

A experiência do Município do Rio de Janeiro com a realização das campanhas para eliminação da sífilis congênita mostrou que essa pode ser uma das estratégias usadas para o enfrentamento do problema. Pode notar-se uma redução de 29,0\% no número de óbitos por sífilis congênita entre os anos de 2000 e 2001, explicado pelos efeitos imediatos das campanhas 15 , com um subseqüente incremento de $39,3 \%$ de 2001 para 2002, quando os esforços de eliminação se encerraram.

A sífilis é causa de prematuridade e de baixo peso ao nascer, e essas duas condições elevam o risco de mortalidade perinatal 27 . As taxas muito elevadas de prematuridade e de baixo peso ao nascer por sífilis congênita encontradas entre os óbitos perinatais ajudam a explicar a sua presença entre as causas mais importantes desses óbitos. Essa constatação só aumenta a necessidade de qualificação da as- 
sistência pré-natal, destacando-se, nesse cenário, o diagnóstico e o tratamento da sífilis materna 28,29 .

Leal \& Szwarcwald 17 (p. 250) já chamavam a atenção para o fato de que, no Estado do Rio de Janeiro entre os anos de 1979 e 1993, nos óbitos no primeiro dia de vida, “... as causas ditas 'reduzíveis por adequado controle da gravidez e adequada atenção ao parto' não demonstraram tendência de decréscimo, permanecendo no mesmo patamar do início dos anos 80". A situação da atenção à gravidez no Município do Rio de Janeiro, em relação à sífilis, não parece ter avançado muito com o passar do tempo.

Como colocam Hartz et al. 30 (p. 312), a mortalidade infantil evitável é "o mais sensível dos indicadores propostos para o evento sentinela da qualidade dos serviços". Os dados deste estudo mostram que ações de vigilância associadas à qualificação do pré-natal e da assistência ao parto, assim como estratégias que permitam o esclarecimento da população em relação à sífilis, podem fazer diferença importante na redução da mortalidade perinatal em nosso meio.

\section{Resumo}

A sífilis permanece como causa importante de mortalidade perinatal no Município do Rio de Janeiro, Brasil, onde o presente estudo foi realizado utilizando os dados do Sistema de Informação de Mortalidade $e$ das Fichas de Notificação e Investigação de Óbitos Fetais e Neonatais, obrigatórias para as maternidades municipais. Entre 1996 e 1998, a sífilis congênita foi responsável por 13,1\% dos óbitos fetais e 6,5\% dos neonatais nas maternidades municipais. Entre 1999 e 2002, os percentuais foram de $16,2 \%$ e 7,9\%, respectivamente. Para o Município do Rio de Janeiro, de 1999 a 2002, os percentuais foram $5,4 \%$ e 2,2\%, para óbitos fetais e neonatais. A taxa de mortalidade perinatal por sífilis congênita permanece estável no Município do Rio de Janeiro apesar dos esforços iniciados com as campanhas para eliminação do agravo em 1999 e 2000. Propomos a utilização da taxa de mortalidade perinatal por sífilis congênita como indicador de impacto das ações de controle e eliminação da sífilis congênita e sugerimos a utilização das fichas de notificação e investigação de óbitos fetais e neonatais para a vigilância de outros agravos evitáveis.

Mortalidade Infantil; Sífilis Congênita; Saúde da Mulher; Bem-Estar da Criança
Os dados aqui apresentados revelam que a mobilização que se pretendia conseguir com as campanhas de eliminação da sífilis congênita, tanto entre profissionais de saúde quanto da população, não foi conseguida, uma vez que, a longo prazo, ações simples e de baixo custo da atenção pré-natal, específicas para prevenir a sífilis congênita, não estão sendo implementadas 15 .

\section{Considerações finais}

Este artigo mostrou que a proposta de monitoramento da mortalidade perinatal específica por sífilis congênita constitui-se em um instrumento efetivo para dimensionar a magnitude do agravo e para orientar as ações de controle e eliminação da sífilis congênita no Município do Rio de Janeiro. O uso das informações advindas da vigilância de óbitos fetais e neonatais pode beneficiar outros componentes das ações programáticas da atenção perinatal, principalmente se essa experiência expandir-se para outros agravos evitáveis.

\section{Colaboradores}

V. Saraceni, M. H. F. S. Guimarães e M. M. Theme Filha participaram da concepção do estudo, da construção e revisão das bases de dados, análise de resultados e construção das tabelas, assim como da revisão da literatura e do manuscrito. M. C. Leal participou da concepção do estudo, da revisão dos resultados e do manuscrito. 


\section{Referências}

1. Mascola L, Pelosi R, Alexander CE. Inadequate treatment of syphilis in pregnancy. Am J Obstet Gynecol 1984; 150:945-7.

2. Hira SK, Bhart G, Chikamata DM, Nkowane B, Tembo G, Perine PL, et al. Syphilis intervention in pregnancy: Zambian demonstration project. Genitourin Med 1990; 66:159-64.

3. Gust DA, Levine WC, St. Louis ME, Braxton J, Berman SM. Mortality associated with congenital syphilis in the United States, 1992-1998. Pediatrics 2002; 109:E79-9.

4. Watson-Jones D, Changalucha J, Gumodoka B, Weiss H, Rusizoka M, Ndeki L, et al. Syphilis in pregnancy in Tanzania. I. Impact of maternal syphilis on outcome of pregnancy. J Infect Dis 2002; 186:940-7.

5. Tikhonova L, Salakhov E, Southwick K, Shakarishvili A, Ryan C, Hillis S, et al. Congenital syphilis in the Russian Federation: magnitude, determinants, and consequences. Sex Transm Infect 2003; 79:106-10

6. Secretaria Municipal de Saúde do Rio de Janeiro. Sífilis na gravidez. Saúde em Foco - Dados 1999; 23.

7. Rouquayrol MZ, Correia LL, Barbosa LM, Xavier LG, Oliveira JW, Fonseca W. Fatores de risco de natimortalidade em Fortaleza: um estudo de caso-controle. J Pediatr (Rio J) 1996; 72:374-8.

8. De Lorenzi DR, Tanaka AC, Bozzetti MC, Ribas FE, Weissheimer L. A natimortalidade como indicador de saúde perinatal. Cad Saúde Pública 2001; 17:141-6.

9. Departamento de Informação e Informática do SUS. Indicadores demográficos básicos 2002. Indicadores de mortalidade. C.2. Taxa de mortalidade perinatal. Brasília: Departamento de Informação e Informática do SUS; 2002.

10. Saraceni V, Nicolai C, Domingues R, Lauria LM, Durovni B, Boaretto MC. Sífilis adquirida e sífilis congênita: ações do Município do Rio de Janeiro. DST J Bras Doenças Sex Transm 2000; 12:95.

11. Organização Mundial da Saúde. Classificação estatística internacional de doenças e problemas relacionados à saúde - 10a revisão. São Paulo: Centro Colaborador da OMS para a Classificação de Doenças em Português; 2001.

12. Rouquayrol MZ, Almeida Filho N. Epidemiologia e saúde. Rio de Janeiro: Medsi; 1999.

13. Wigglesworth J. Monitoring perinatal mortality. A pathophysiological approach. Lancet 1980; 2:684-6.

14. World Health Organization. International classification of diseases. Manual of the international statistical classification of diseases, injuries and causes of death. Geneva: World Health Organization; 1977.

15. Saraceni V, Leal MC. Avaliação da efetividade das campanhas para eliminação da sífilis congênita na redução da morbi-mortalidade perinatal. Município do Rio de Janeiro, 1999-2000. Cad Saúde Pública 2003; 19:1341-9.

16. Lago EG, Rodrigues L, Fiori RM, Stein AT. Congenital syphilis: identification of two distinct profiles of maternal characteristics associated with risk. Sex Transm Dis 2004; 31:33-7.
17. Leal MC, Szwarcwald CL. Evolução da mortalidade neonatal no Estado do Rio de Janeiro, Brasil (1979-1993): análise por causa segundo grupo de idade e região de residência. Cad Saúde Pública 1996; 12:243-52.

18. Aquino MM, Cecatti JG, Mariani Neto C. Risk factors associated to fetal death. Rev Paul Med 1998; 116:1852-7.

19. Labbe AC, Mendonça AP, Alves AC, Jaffar S, Dias F, Alvarenga IC, et al. The impact of syphilis, HIV1 , and HIV-2 on pregnancy outcome in Bissau, Guinea-Bissau. Sex Transm Dis 2002; 29:157-67.

20. Folgosa E, Osman NB, Gonzalez C, Hagerstrand I, Bergstrom S, Ljungh A. Syphilis seroprevalence among pregnant women and its role as a risk factor for stillbirth in Maputo, Mozambique. Genitourin Med 1996; 72:339-42.

21. Watts TE, Larsen SA, Brown ST. A case-control study of stillbirths at a teaching hospital in Zambia, 1979-80: serological investigations for selected infectious agents. Bull World Health Organ 1984; 62:803-8.

22. Szwarcwald CL, Leal MC, Andrade CL, Souza Jr. PR. Estimação da mortalidade infantil no Brasil: o que dizem as informações sobre óbitos e nascimentos do Ministério da Saúde? Cad Saúde Pública 2002; 18:1725-36.

23. Carrara S. Tributo à Vênus: a luta contra a sífilis no Brasil, da passagem do século aos anos 40. Rio de Janeiro: Editora Fiocruz; 1996.

24. Lansky S, França E, Leal MC. Mortalidade perinatal e evitabilidade: revisão da literatura. Rev Saúde Pública 2002; 36:759-72.

25. Leal MC, Gama SGN, Campos MR, Cavalini LC, Garbayo LC, Brasil CLP, et al. Fatores associados à morbi-mortalidade perinatal em uma amostra de maternidades públicas e privadas do Município do Rio de Janeiro, 1999-2001. Cad Saúde Pública 2004; 20 Suppl 1:S20-33.

26. Gomes MASM. Aspectos da qualidade do atendimento à gestação e ao parto através da percepção das usuárias [Dissertação de Mestrado]. Rio de Janeiro: Fundação Oswaldo Cruz; 1995.

27. Victora CG, Barros F, Vaughan JP. Epidemiologia da desigualdade. São Paulo: Editora Hucitec; 1988.

28. Conde-Agudelo A, Belizan JM, Diaz-Rossello JL. Epidemiology of fetal death in Latin America. Acta Obstet Gynecol Scand 2000; 79:371-8.

29. Liljestrand J. Reducing perinatal and maternal mortality in the world: the major challenges. Br J Obstet Gynaecol 1999; 106:877-80.

30. Hartz ZM, Champagne F, Leal MC, Contandriopoulos AP. Mortalidade infantil "evitável" em duas cidades do Nordeste do Brasil: indicador de qualidade do sistema local de saúde. Rev Saúde Pública 1996; 30:310-8.

Recebido em 07/Jul/2004

Versão final reapresentada em 13/Out/2004 Aprovado em 07/Mar/2005 